\title{
Deadbeat Source Localization from Range-only Measurements: a Robust Kernel-based Approach
}

\author{
Peng Li, Gilberto Pin, Giuseppe Fedele, and Thomas Parisini
}

\begin{abstract}
This paper presents a novel framework for the problem of target localization based on the range information collected by a single mobile agent. The proposed methodology exploits the algebra of Volterra integral operators to annihilate the influence of initial conditions on the transient phase, thus achieving a deadbeat performance. The robustness properties against additive measurement perturbations are analyzed and the bias caused by the time-discretization is characterized as well. Extensive simulation results and comparisons are provided showing the effectiveness of the proposed technique in coping with both stationary and drifting targets.
\end{abstract}

\section{INTRODUCTION}

The problem of target localization represents one of the fundamental issues in several engineering fields such as aerospace, military, wireless sensor networks, etc. Several methods and tools have been developed targeting specific applications with customized system architectures and algorithmic solutions. Many recent works can be found in the literature and a comprehensive survey is out of the scope of the paper. Therefore, in the following only a partial overview will be given. For an expanded literature review, the reader is referred, for instance, to the important recent paper [1].

The localization systems can be classified into two categories: the global positioning systems (GPS) and the local positioning system (LPS) [2]. GPS is the most obvious and easy solution to the localization problem in an open environment. However, due to the limited visibility of the satellites, there are practical situations in which GPS cannot be used [3].

In the context of the local positioning for target pursuing, usually it is possible to acquire only partial data related to the coordinates of the target, such as distance, angle and bears [4], which act as clues of the estimation. In this work, only distance-based methods will be considered. Notably, the

This work has been partially supported by the European Union's Horizon 2020 research and innovation programme under grant agreement No 739551 (KIOS CoE).

P. Li is with the Dept. of Electrical and Electronic Engineering, Imperial College London, UK peng. li13@imperial.ac.uk

G. Pin is with Electrolux Italia S.p.A, Italy gilbertopindalice. it

G. Fedele is with the Department of Informatics, Modeling, Electronics and Systems Engineering, University of Calabria, Italy giuseppe. fedeledunical.it

T. Parisini is with the Dept. of Electrical and Electronic Engineering at the Imperial College London, UK, with the KIOS Research and Innovation Centre of Excellence, University of Cyprus, and also with the Dept. of Engineering and Architecture at the University of Trieste, Italy (t.parisini@gmail.com) distance-based localization problem, also known as rangebased, has been widely investigated together with its application to the case of underwater vehicles navigation (see [5], [6], [7]). Among other range-based localization methods, the algorithms proposed in [8], [9] calculate the coordinate of the target by fusing the data collected by multiple agents. All agents measure range information at the same time, and the location is calculated off-line by exploiting geometric constraints. These algorithms are not very well suited for realtime target tracking problems and are sometimes not robust with respect to the noise. Instead, continuous-time on-line localization algorithms may outperform the off-line ones in terms of robustness and tracking performance. In the literature, a typical family of algorithms is the one in which multiple distributed sensing agents cooperate in order to estimate the source location (see, for instance, [10], [11]). These algorithms are often characterized by the need of synchronization tools to achieve consensus on the target location, at the cost of an increased computational burden and stringent requirements on the communication network.

Taking also computational and infrastructural complexity into consideration, several single-moving-agent localization systems have been proposed. In [12], 2D single-range-base localization is formulated as a nonlinear system whose observability is studied resorting to an algebraic derivative estimator. A single-agent 3D localization method is presented in [13] and [14], where the position of the source together with its velocity are regarded as the hidden states of a nonlinear system. To estimate the states, the nonlinear system is transformed into a linear time-varying (LTV) one. After a further transformation, the LTV system is converted into a linear time invariant (LTI) one, to which general-purpose state observers, e.g., Luenberger, Kalman or $H_{\infty}$ filters can be applied in order to estimate the states. However, the computational burden is significant. Moreover, the measurement of velocity is required introducing additional difficulties of implementation and possibly extra measurement errors. In [1], an alternative LTI system with time-varying output mapping is formulated with comprehensive observability analysis. Moreover, a robust correctionbased state estimator is designed with the consideration of disturbances on both the distance measurement and velocity.

Another family of approaches is based on regression formulation derived from geometrical relationship (see [15], [16], [17], [18]). Specifically, [15] presents a continuous-time adaptive algorithm that is able to localize the target, which can be either stationary or drifting. A set of linear filters is used here to avoid the explicit calculation of the signal derivatives in the differential equation. A similar approach can be found also in [16]. Based on the aforementioned 
methods, [17] presents a least-square-based adaptive law with a forgetting factor aiming at minimizing the cost function with respect to the target location, which is proven to be superior to the gradient algorithms due to higher convergence rate and robustness. A discrete-time localization scheme with fading memory is proposed in [18] with a velocity-related identifiability condition guarantee the non-singularity of the regression matrix.

All the above-mentioned approaches are shown to enjoy exponentially stable convergence properties. However, in many practical applications characterized by strict requirements on the tracking speed, it is often desirable that the estimation converge to the true location in finite-time. A novel kernelbased approach is first proposed in [19] providing a deadbeat parametric estimation method for continuous-time linear systems. By using Volterra integral operators, this method allows to annihilate the effects of the unknown initial conditions of the states. Volterra integral operators with a specific kernel have been also used in [20] to obtain a finite-time convergent observer for continuous-time SISO linear systems.

Besides the aforementioned linear estimation frameworks, in our recent work [21], Volterra integral operators have been applied to the nonlinear range-based localization problem, yielding the position estimation of a fixed target in a deadbeat manner in the ideal case. As an extension of this earlier work, the present paper deals not only with the stationary target but also with the localization problem in the presence of a persistent drift on the target. Notably, the robust stability analysis provides evidence that the estimation error remains bounded in case of a persistently drifting target and measurement noise, making the proposed algorithm a viable choice for the practical implementation.

This paper is organized as follows. In the next section, the localization problem is stated. In Section III some preliminaries of the Volterra operator and the core properties of non-asymptotic kernels are given. Section IV formulates the continuous-time non-asymptotic algorithm based on the Volterra operators. Section V analyzes the robustness of the proposed algorithm under additive bounded noise, as well as the characterization of the bias introduced by the discrete implementation. In Section VI, the algorithm is shown to be able to track a source drifting within a bounded range and with bounded rate. Finally, Section VII presents numerical results and comparisons with another continuous-time fast-converging localization method in [17] showing the effectiveness of the proposed algorithm.

\section{Problem Statement AND Preliminaries}

Consider a target positioned in a three-dimensional coordinate system, the position of which is described in the form of a vector $\boldsymbol{x} \in \mathbb{R}^{3}$. A mobile agent, whose coordinates at time $t$ is also a vector $\boldsymbol{y}(t) \in \mathbb{R}^{3}$, is moving according to a preset trajectory and measuring, for any $t \geq 0$, the distance from the target. The source location $\boldsymbol{x}$ can be estimated based on the knowledge of the agent position $\boldsymbol{y}(t)$ and the measured distance $d(t)$ according to:

$$
d(t)=\|\boldsymbol{y}(t)-\boldsymbol{x}\|
$$

where $\|\cdot\|$ denotes the Euclidean norm. We make the following assumption on the boundedness of position, velocity and acceleration of the agent [15].

Assumption 1: The agent trajectory described by the function $\boldsymbol{y}(t)$ is at least twice differentiable. Moreover the agent's trajectory function $\boldsymbol{y}(t)$ and its velocity $\frac{d}{d t} \boldsymbol{y}(t)$ are bounded, i.e. $\forall t \geq 0$, there exists positive $M_{i}(i=1,2)$, such that

$$
\begin{gathered}
\|\boldsymbol{y}(t)\| \leq M_{1}, \\
\left\|\frac{d}{d t} \boldsymbol{y}(t)\right\| \leq M_{2} .
\end{gathered}
$$

Notice that (1) is a scalar algebraic constraint which is not sufficient to retrieve the position of the agent. In order to retrieve an estimate of the agents's position by using the kernel-based method, we need to transform (1) in a differential constraint and augment the scalar equation with further dynamic equations.

Taking the first derivative of (1), one can obtain

$$
\frac{d}{d t}\left\{d^{2}(t)\right\}=2 \frac{d}{d t} \boldsymbol{y}(t)^{\top}(\boldsymbol{y}(t)-\boldsymbol{x}) .
$$

Defining the signals

$$
\begin{aligned}
& s_{d}(t) \triangleq d^{2}(t), \\
& s_{y}(t) \triangleq \boldsymbol{y}(t)^{\top} \boldsymbol{y}(t),
\end{aligned}
$$

one can rewrite (4) as

$$
\frac{d}{d t}\left\{s_{d}(t)\right\}=\frac{d}{d t}\left\{s_{y}(t)\right\}-2 \frac{d}{d t}\left\{\boldsymbol{y}(t)^{\top}\right\} \boldsymbol{x}
$$

However, the only available quantities in (7) are $d(\cdot), \boldsymbol{y}(\cdot)$, $s_{d}(\cdot)$ and $s_{y}(\cdot)$, while their derivatives are assumed not directly accessible.

\section{NON-ASYMPtOTIC VolterRa Operators Algebra}

In the proposed approach, Volterra integral operators play a key role in transforming a differential expression into a sequence of algebraic equations. Basic concepts and algebra of the Volterra operators (see [19], [22] and the reference therein) are briefly reviewed and some notations are introduced in this section for the reader's convenience.

Let $V_{K}$ denote the bounded Volterra linear integral operator induced by a given bivariate kernel function $K(\cdot, \cdot)$ mapping within the Hilbert space $\mathcal{L}_{\text {loc }}^{2}\left(\mathbb{R}_{\geq 0}\right)$ of locally square-integrable functions with domain $\mathbb{R}_{\geq 0}$ and range $\mathbb{R} .{ }^{1}$. Then, a function $u(\cdot) \in \mathcal{L}_{\text {loc }}^{2}\left(\mathbb{R}_{\geq 0}\right)$ has the image function $\left[V_{K} u\right](\cdot)$ through $V_{K}$ defined as

$$
\left[V_{K} u\right](t) \triangleq \int_{0}^{t} K(t, \tau) u(\tau) d \tau,
$$

where the kernel function $K(\cdot, \cdot): \mathbb{R} \times \mathbb{R} \rightarrow \mathbb{R}$ is taken as a Hilbert-Schmidt $(\mathcal{H S})$ Kernel Function. The notation $u^{(i)}(t)$

\footnotetext{
${ }^{1}$ For a given positive integer $i$ and a function $u(\cdot)$, we introduce the following definition: $\left(u(\cdot) \in \mathcal{L}_{\text {loc }}^{i}\left(\mathbb{R}_{\geq 0}\right) \Leftrightarrow\left(u(\cdot): \mathbb{R}_{\geq 0} \rightarrow \mathbb{R}\right) \wedge\left(\int_{B}|u(t)|^{i} d t<\right.\right.$ $\infty, \forall$ compact $B \subset \mathbb{R}_{\geq 0}$ )
} 
will be used to address the $i$-th weak derivative of a function $u(t)$ defined as:

Definition 3.1: (Weak (generalized) derivative): Let $u(\cdot) \in$ $\mathcal{L}_{\text {loc }}^{1}\left(\mathbb{R}_{\geq 0}\right)$. Then $u^{(1)}(\cdot)$ is said to be a weak derivative of $u(\cdot)$ if

$$
\int_{0}^{t} u(\tau)\left(\frac{d}{d \tau} \phi(\tau)\right) d \tau=-\int_{0}^{t} u^{(1)}(\tau) \phi(\tau) d \tau, \forall t \in \mathbb{R}_{\geq 0}
$$

for all $\phi \in \mathcal{C}^{\infty}$, with $\phi(0)=\phi(t)=0$.

\section{A. Dynamic implementation of Volterra operators}

To realise the transformation of the signal $u(t)$ through the Volterra operator $V_{K}$, a dynamic system having $\left[V_{K} u\right](t)$ as output can be constructed, for $t \geq 0$, as follows:

$$
\begin{aligned}
& \left\{\begin{array}{c}
\xi^{(1)}(t)=K(t, t) u(t)+\int_{0}^{t}\left(\frac{\partial}{\partial t} K(t, \tau)\right) u(\tau) d \tau \\
\xi(0)=\xi_{0}=\int_{0}^{0} K(0, \tau) u(\tau) d \tau=0
\end{array},\right. \\
& {\left[V_{K} u\right](t)=\xi(t),}
\end{aligned}
$$

where $\xi^{(1)}(t)=\frac{d}{d t}\left[V_{K} u\right](t)$ which is obtained according to the Leibnitz integral rule.

By considering the image function of the time-derivatives of the signal, the following characteristic can be derived by applying integration by parts.

Lemma 3.1: Given an $i \in \mathbb{Z}_{>0}$, exploiting a signal $u(\cdot) \in$ $\mathcal{L}_{\text {loc }}^{2}\left(\mathbb{R}_{\geq 0}\right)$ and a Volterra kernel function $K(\cdot, \cdot) \in \mathcal{H S}$ which is at least $i$-th order differentiable with respective to the second argument, it holds that:

$$
\begin{aligned}
{\left[V_{K} u^{(i)}\right](t) } & =\sum_{j=0}^{i-1}(-1)^{i-j-1} u^{(j)}(t) K^{(i-j-1)}(t, t) \\
& +\sum_{j=0}^{i-1}(-1)^{i-j} u^{(j)}(0) K^{(i-j-1)}(t, 0) \\
& +(-1)^{i}\left[V_{K^{(i)}} u\right](t), \quad \forall t \in \mathbb{R}_{\geq 0} .
\end{aligned}
$$

Lemma 3.1 indicates the non-anticipativity of the image function $\left[V_{K} u^{(i)}\right](t)$ with respect to the signal $u(\cdot)$ and its lower-order derivatives $u^{(1)}(\cdot), u^{(2)}(\cdot), \ldots, u^{(i-1)}(\cdot)$.

\section{B. Kernel conditions for non-asymptoticity}

Notice that, in general, terms of initial conditions $u(0)$, $u^{(1)}(0), \ldots, u^{(i)}(0)$ are included in (9) when the auxiliary signal $\left[V_{K} u^{(i)}\right](t)$ is computed. The influence of the unknown initial conditions is one of the factors that affect the convergence of the estimation and theoretically avoids the possibility to obtain a deadbeat performance in noise-free scenarios.

In this paper, the finite-time convergence will be realized by rendering the transformed signal $\left[V_{K} u^{(i)}\right](t)$ independent from the unknown initial states $u(0), u^{(1)}(0), \ldots, u^{(i)}(0)$, for all $t \in \mathbb{R}_{\geq 0}$, by using kernel functions obeying the following definition:
Definition 3.2: ( $i$-th Order Non-asymptotic Kernel): Consider a Volterra kernel function that subsumes the assumption in Lemma 3.1, and for given $i \in \mathbb{Z}_{\geq 0}$, it verifies the condition

$$
K^{(j)}(t, 0)=0, \forall t \in \mathbb{R}_{\geq 0}, \forall j \in\{0, \ldots, i-1\},
$$

then $K(\cdot, \cdot)$ is called an $i$-th order non-asymptotic kernel.

In this work, we exploit a kernel $K(\cdot, \cdot)$, satisfying more stringent conditions, for given $i \geq 1$

$$
\left\{\begin{array}{l}
K^{(j)}(t, 0)=0 \\
K^{(j)}(t, t)=0
\end{array}\right.
$$

$\forall t \in \mathbb{R}_{\geq 0}$ and $\forall j \in\{0, \ldots, i-1\}$.

It can be immediately proved that the image of signal's derivatives no longer depend on the current value of the derivatives of $u(\cdot)$ and (9) reduces to the simple expression:

$$
\left[V_{K} u^{(i)}\right](t)=(-1)^{i}\left[V_{K^{(i)}} u\right](t) .
$$

\section{A KERNEL-BASED NON-ASYMPTOTIC LOCALIZATION ALGORITHM}

In this section, the deadbeat localization algorithm is presented.

Taking the transformation on both sides of the equation through the Volterra operator (7), we will immediately obtain:

$$
\left[V_{K} s_{d}{ }^{(1)}\right](t)=\left[V_{K} s_{y}{ }^{(1)}\right](t)-2\left[\boldsymbol{V}_{K} \boldsymbol{y}^{(1)}\right](t)^{\top} \boldsymbol{x},
$$

which, according to (10), is equivalent to

$$
\left[V_{K^{(1)}} s_{d}\right](t)=\left[V_{K^{(1)}} s_{y}\right](t)-2\left[\boldsymbol{V}_{\boldsymbol{K}^{(1)}} \boldsymbol{y}\right](t)^{\top} \boldsymbol{x} .
$$

Please note that (12) no longer relies on the derivatives of the measurements which cannot be exactly obtained due to the unknown initial conditions, but relies on the derivatives of the known kernel functions. Therefore, without the effect of the unknown initial conditions, deadbeat estimation can be achieved by solving the following linear equation for $\boldsymbol{x}$ :

$$
r(t)=\boldsymbol{z}(t)^{\top} \boldsymbol{x},
$$

where we have used the notation

$$
\begin{aligned}
r(t) & =\frac{-\left[V_{K^{(1)}} s_{d}\right](t)+\left[V_{K^{(1)}} s_{y}\right](t)}{2}, \\
\boldsymbol{z}(t) & =\left[\boldsymbol{V}_{\boldsymbol{K}^{(1)}} \boldsymbol{y}\right](t) .
\end{aligned}
$$

Since the highest order of derivatives involved in the linear differential equation (7) is one, then kernel functions with order equal or higher than one are adequate to perform a nonasymptotic identification. Therefore, as in [19], we choose the following kernel function:

$$
K(t, \tau) \triangleq e^{-\omega(t-\tau)}\left(1-e^{-\omega \tau}\right)\left[1-e^{-\omega(t-\tau)}\right] .
$$

In (15), the factor $\left(1-e^{-\omega(t-\tau)}\right)$ guarantees the causality of the kernel function while the non-asymptotic condition is realized by the term $\left(1-e^{-\omega \tau}\right)$. Both the factors contribute to the boundedness of the kernel function.

From now on, we are presenting how the augmentation works mapping the first order derivatives $s_{y}^{(1)}(t), s_{d}^{(1)}(t)$ and 
$\boldsymbol{y}^{(1)}(t)$ to the auxiliary signals $\left[V_{K^{(1)}} s_{y}\right](t),\left[V_{K^{(1)}} s_{d}\right](t)$ and $\left[\boldsymbol{V}_{\boldsymbol{K}^{(1)}} \boldsymbol{y}\right](t)$. Taking the first derivative of the kernel function (15) with respect to the second argument $\tau$, we get

$$
K^{(1)}(t, \tau)=e^{-\omega t} f_{\omega, 1}(\tau)+e^{-2 \omega t} f_{\omega, 2}(\tau),
$$

where

$$
\begin{aligned}
& f_{\omega, 1}(\tau)=\omega e^{\omega \tau}, \\
& f_{\omega, 2}(\tau)=\omega e^{\omega \tau}-2 \omega e^{2 \omega \tau} .
\end{aligned}
$$

Define

$$
K_{j}(t, \tau) \triangleq e^{-\omega j t} f_{\omega, j}(\tau), \quad j=1,2 .
$$

Due to the linearity of Volterra operators, it holds that

$$
\left[V_{K^{(1)}} u\right](t)=\left[V_{K_{1}} u\right](t)+\left[V_{K_{2}} u\right](t) .
$$

Noticing that

$$
\frac{\partial}{\partial t} K_{j}(t, \tau)=-\omega j e^{-\omega j t} f_{\omega, j}(\tau)=-j \omega K_{j}(t, \tau),
$$

and letting $\xi_{u, j}(t) \triangleq\left[V_{K_{j}} u\right](t)$, where $u$ represents the signals $s_{d}(t), s_{y}(t)$ and $\boldsymbol{y}(t)$ with $j \in\{1,2\}$, it follows that the auxiliary signal vector can be expressed as:

$$
\boldsymbol{z}_{e}(t)=\left[\left[V_{K^{(1)}} s_{d}\right](t),\left[V_{K^{(1)}} s_{y}\right](t),\left[\boldsymbol{V}_{\boldsymbol{K}^{(1)}} \boldsymbol{y}\right](t)^{\top}\right]^{\top},
$$

whose elements are needed to form $r(t)$ and $\boldsymbol{z}(t)$ in the constraint (13). The elements of the vector $\boldsymbol{z}_{e}(t)$ can be expressed as the output of a 10-dimensional linear time-varying dynamic system

$$
\left\{\begin{array}{l}
\boldsymbol{\xi}^{(1)}(t)=\boldsymbol{G}_{\xi} \boldsymbol{\xi}(t)+\boldsymbol{E}_{\xi}(t) \boldsymbol{u}_{\xi}(t), \\
\boldsymbol{z}_{e}(t)=\boldsymbol{H} \boldsymbol{\xi}(t)
\end{array}\right.
$$

where $\boldsymbol{\xi}(t) \in \mathbb{R}^{10}$ is the overall state vector and $\boldsymbol{\xi}(0)=\mathbf{0}$ with

$$
\begin{aligned}
\boldsymbol{\xi}(t) & =\left[\xi_{s_{d}, 1}(t), \xi_{s_{d}, 2}(t), \xi_{s_{y}, 1}(t), \ldots \xi_{y_{3}, 1}(t), \xi_{y_{3}, 2}(t)\right]^{\top}, \\
\boldsymbol{u}_{\xi}(t) & =\boldsymbol{u}_{a}(t) \otimes[1,1]^{\top} \in \mathbb{R}^{10}, \\
\boldsymbol{u}_{a}(t) & =\left[s_{d}(t), s_{y}(t), \boldsymbol{y}(t)\right]^{\top} \in \mathbb{R}^{5}, \\
\boldsymbol{G}_{\xi} & =\operatorname{blockdiag}(\boldsymbol{G}, \boldsymbol{G}, \boldsymbol{G}, \boldsymbol{G}, \boldsymbol{G}), \\
\boldsymbol{G} & =\operatorname{diag}(-\omega,-2 \omega), \\
\boldsymbol{E}_{\xi}(t) & =\operatorname{blockdiag}(\boldsymbol{E}(t), \boldsymbol{E}(t), \boldsymbol{E}(t), \boldsymbol{E}(t), \boldsymbol{E}(t)), \\
\boldsymbol{E}(t) & =\operatorname{diag}\left(K_{1}(t, t), K_{2}(t, t)\right), \\
\boldsymbol{H} & =\operatorname{blockdiag}\left(\mathbf{1}^{\top}, \mathbf{1}^{\top}, \mathbf{1}^{\top}, \mathbf{1}^{\top}, \mathbf{1}^{\top}\right), \\
\mathbf{1}^{\top} & \in \mathbb{R}^{1 \times 2}
\end{aligned}
$$

with $\otimes$ denoting Kronecker product.

Up to now, all the values of the signals in the linear constraint (13) are available thanks to (18).

By defining

$$
\begin{aligned}
& \boldsymbol{S}(t) \triangleq \boldsymbol{z}(t) r(t) \in \mathbb{R}^{3 \times 1}, \\
& \boldsymbol{R}(t) \triangleq \boldsymbol{z}(t) \boldsymbol{z}(t)^{\top} \in \mathbb{R}^{3 \times 3},
\end{aligned}
$$

the problem becomes that of estimating $\boldsymbol{x}$ from:

$$
\boldsymbol{S}(t)=\boldsymbol{R}(t) \boldsymbol{x} .
$$

Noticing that $\operatorname{rank}[\boldsymbol{R}(t)]=1$, it follows that the above equation cannot be directly solved for $x$. To address this issue, we resort to a filtered version of (21) which holds for all $t \geq 0$. Applying to both sides of (21) another Volterra operator $V_{G}$, with a simple kernel $G(t, \tau)=e^{-g(t-\tau)}, g \in \mathbb{R}_{>0}$, we have

$$
\left[\boldsymbol{V}_{\boldsymbol{G}} \boldsymbol{S}\right](t)=\left[\boldsymbol{V}_{\boldsymbol{G}} \boldsymbol{R}\right](t) \boldsymbol{x}, \forall t \in \mathbb{R}_{\geq 0} .
$$

Denoting $\boldsymbol{S}_{f}(t) \triangleq\left[\boldsymbol{V}_{\boldsymbol{G}} \boldsymbol{S}\right](t)$ and $\boldsymbol{R}_{f}(t) \triangleq\left[\boldsymbol{V}_{\boldsymbol{G}} \boldsymbol{R}\right](t)$, it follows that

$$
\left\{\begin{array}{l}
\dot{\boldsymbol{S}}_{f}(t)=-g \boldsymbol{S}_{f}(t)+\boldsymbol{S}(t) \\
\dot{\boldsymbol{R}}_{f}(t)=-g \boldsymbol{R}_{f}(t)+\boldsymbol{R}(t)
\end{array},\right.
$$

where $\boldsymbol{S}_{f}(0)=\mathbf{0}_{3 \times 1}, \boldsymbol{R}_{f}(0)=\mathbf{0}_{3 \times 3}$.

Remark 4.1: Thanks to the exponential shape of the kernel $G(t, \tau)$, the covariance filtering introduces a fading memory mechanism. By tuning the forgetting factor $g$, the dependence on the previous data can be adjusted, which allows the localization scheme to face measurement noise and source drift. The interested readers can get a deeper insight on how $g$ affects the performance of the estimator in [19].

In order to prove the stability of the estimator, we introduce the following Assumption which ensures the invertibility of the filtered covariance matrix $\boldsymbol{R}_{f}(t)$.

Assumption 2: (Persistency of Excitation) The norm of $z(t)$ is persistently exciting (PE) in the sense that there exists constant $\alpha>0$ and $t_{\alpha}>0$ such that $\forall t \geq t_{\alpha}$

$$
\int_{t-t_{\alpha}}^{t} \boldsymbol{z}(\tau) \boldsymbol{z}(\tau)^{\top} d \tau \geq \alpha \boldsymbol{I}>0
$$

Therefore, the non-singularity of $R_{f}(t)$ can be guaranteed due to the fact that $\forall t \geq t_{\alpha}$

$$
\begin{aligned}
\boldsymbol{R}_{f}(t) & \geq \int_{t-t_{\alpha}}^{t} e^{-g(t-\tau)} \boldsymbol{z}(\tau) \boldsymbol{z}(\tau)^{\top} d \tau \\
& \geq e^{-g t_{\alpha}} \int_{t}^{t+t_{\alpha}} \boldsymbol{z}(\tau) \boldsymbol{z}(\tau)^{\top} d \tau \geq e^{-g t_{\alpha}} \alpha I .
\end{aligned}
$$

In this case, the filtered auto-covariance matrix $\boldsymbol{R}_{f}(t)$ characterizes a sufficiently informative output signal at time $t \geq t_{\alpha}$, such that an estimate $\hat{x}(t)$ of the location can be obtained by

$$
\hat{\boldsymbol{x}}(t)=\boldsymbol{R}_{f}(t)^{-1} \boldsymbol{S}_{f}(t) .
$$

Moreover, to avoid the large overshoot in the initial phase of estimation which is caused by inverting a nearly singular matrix $\boldsymbol{R}_{f}(t)$, the inversion in (25) will be performed only when the smallest singular value of $\boldsymbol{R}_{f}(t)$ is greater than a given threshold $\bar{\theta}>0$, to be chosen according to the required numerical accuracy.

Remark 4.2: The P.E. condition (see (24)) depends only upon the trajectory of the sensing agent $\boldsymbol{y}(t)$, which is predetermined. Moreover, recall the definition of $\boldsymbol{z}(t)$ in (14) and (10), condition in (24) is equivalent to there exist $\sigma>0$ and $t_{\sigma}>0$,

$$
\int_{t-t_{\sigma}}^{t} \frac{d}{d t} \boldsymbol{y}(\tau) \frac{d}{d t} \boldsymbol{y}(\tau)^{\top} d \tau \geq \sigma I, \forall t>t_{\sigma} .
$$


Therefore in order to guarantee persistency of excitation, the motion of the agent should span the 3D space.

Remarkably, thanks to the linearity of the Volterra integral operators and recalling the equivalence between (11) and (12), the estimates $\hat{\boldsymbol{x}}(t)$ provided by (25) will converge, nonasymptotically, to the true source position $\boldsymbol{x}$ as long as the persistency of excitation condition (24) or (26) is satisfied.

\section{RobUSTNESS ANALYSIS}

In this section, the performance of the algorithm in presence of bounded measurement perturbations is analyzed and, moreover, the bias introduced by time-discretization in implementation is addressed.

\section{A. Stability analysis}

In order to characterize the robustness of the algorithm in facing measurement perturbations, the stability analysis is performed under the assumption that both $d(t)$ and $\boldsymbol{y}(t)$ are affected by bounded additive disturbances:

$$
\begin{aligned}
\hat{d}(t) & =d(t)+\eta_{d}(t), \\
\hat{\boldsymbol{y}}(t) & =\boldsymbol{y}(t)+\boldsymbol{\eta}_{y}(t),
\end{aligned}
$$

where $\left|\eta_{d}(t)\right| \leq \bar{\eta}_{d}, \boldsymbol{\eta}_{y}=\left[\eta_{y, 1}, \eta_{y, 2}, \eta_{y, 3}\right]^{\top}$ and $\left|\boldsymbol{\eta}_{y}(t)\right| \leq \overline{\boldsymbol{\eta}}_{y}$.

Recalling (18) and accounting for the exogenous perturbation we get:

$$
\hat{\boldsymbol{\xi}}^{(1)}(t)=\boldsymbol{G}_{\xi} \hat{\boldsymbol{\xi}}(t)+\boldsymbol{E}_{\xi}\left(\boldsymbol{u}_{\xi}(t)+\boldsymbol{\eta}(t)\right),
$$

where $\boldsymbol{\eta}(t)=\left[\eta_{s d}(t), \eta_{s d}(t), \eta_{s y}(t), \eta_{s y}(t), \boldsymbol{\eta}_{y}(t) \otimes[1,1]\right]^{\top}$ is a column vector with 10 entries with definitions

$$
\begin{aligned}
\eta_{s d}(t) & \triangleq 2 d \eta_{d}+\eta_{d}^{2} \\
& \leq 2\left(M_{1}+\|x\|\right) \bar{\eta}_{d}+\bar{\eta}_{d}^{2} \triangleq \bar{\eta}_{1}=\bar{\eta}_{2} . \\
\eta_{s y}(t) & \triangleq 2 y_{1} \eta_{y, 1}+\eta_{y, 1}^{2}+2 y_{2} \eta_{y, 2}+\eta_{y, 2}^{2}+2 y_{3} \eta_{y, 3}+\eta_{y, 3}^{2} \\
& \leq 6 M_{1} \bar{\eta}_{d}+3 \bar{\eta}_{d}^{2} \triangleq \bar{\eta}_{3}=\bar{\eta}_{4} .
\end{aligned}
$$

The dynamics of the state error $\tilde{\boldsymbol{\xi}}(t)=\hat{\boldsymbol{\xi}}(t)-\boldsymbol{\xi}(t)$ can be expressed as:

$$
\tilde{\boldsymbol{\xi}}^{(1)}(t)=\boldsymbol{G}_{\xi} \tilde{\boldsymbol{\xi}}(t)+\boldsymbol{E}_{\xi} \boldsymbol{\eta}(t) .
$$

Since the matrix $\boldsymbol{G}_{\xi}$ is Hurwitz and $\boldsymbol{E}_{\xi} \boldsymbol{\eta}(t)$ is bounded, then $\tilde{\boldsymbol{\xi}}(t)$ is bounded. Each element of the error vector $\tilde{\boldsymbol{\xi}}(t) \triangleq\left[\tilde{\xi}_{1}(t), \tilde{\xi}_{2}(t), \ldots \tilde{\xi}_{10}(t)\right]^{\top}$, verifies the norm-inequality:

$$
\begin{aligned}
\left|\tilde{\xi}_{2 i+k}(t)\right| \leq & e^{-k \omega t} \tilde{\xi}_{2 i+k}(0) \\
& +\frac{1-e^{-k \omega t}}{k \omega} \sup _{0 \leq \tau \leq t} K_{k}(\tau, \tau) \bar{\eta}_{2 i+k} \\
\leq & e^{-k \omega t} \tilde{\xi}_{2 i+k}(0) \\
& +\frac{1}{k \omega} \sup _{0 \leq \tau \leq t} K_{k}(\tau, \tau) \bar{\eta}_{2 i+k} \\
\triangleq & \overline{\tilde{\xi}}_{2 i+k}, \forall i \in\{0,1,2,3,4\}, k \in\{1,2\}
\end{aligned}
$$

where $\bar{\eta}_{i}$ represents the upper bound of the $i$-th element of the disturbance vector $\boldsymbol{\eta}(t)$.
Therefore, the auxiliary-signal error $z_{e}$ due to the measurement disturbances:

$$
\tilde{\boldsymbol{z}}_{e}(t)=\boldsymbol{H} \tilde{\boldsymbol{\xi}}(t)
$$

is bounded, too.

It follows that the error on $r(t)$ and $\boldsymbol{z}(t)$ in (14) in turn verifies the inequalities:

$$
\begin{aligned}
|\tilde{r}(t)| & \leq \frac{\overline{\tilde{\xi}}_{3}+\overline{\tilde{\xi}}_{4}+\overline{\tilde{\xi}}_{1}+\overline{\tilde{\xi}}_{2}}{2}, \\
|\tilde{\boldsymbol{z}}(t)| & \leq\left[\overline{\tilde{\xi}}_{5}+\overline{\tilde{\xi}}_{6}, \overline{\tilde{\xi}}_{7}+\overline{\tilde{\xi}}_{8}, \overline{\tilde{\xi}}_{9}+\overline{\tilde{\xi}}_{10}\right]^{\top},
\end{aligned}
$$

that imply the boundedness of $\tilde{r}(t)$ and $\tilde{\boldsymbol{z}}(t)$, as well as of $\hat{r}(t)$ and $\hat{z}(t)$.

Moreover, according to the definition (20), one can obtain

$$
\begin{aligned}
& |\hat{\boldsymbol{S}}(t)| \in\left[\inf _{0 \leq \tau \leq t} \hat{\boldsymbol{z}}(t) \hat{r}(t), \sup _{0 \leq \tau \leq t} \hat{\boldsymbol{z}}(t) \hat{r}(t)\right], \\
& |\hat{\boldsymbol{R}}(t)| \in\left[\inf _{0 \leq \tau \leq t} \hat{\boldsymbol{z}}(t) \hat{\boldsymbol{z}}(t)^{\top}, \sup _{0 \leq \tau \leq t} \hat{\boldsymbol{z}}(t) \hat{\boldsymbol{z}}(t)^{\top}\right] .
\end{aligned}
$$

The proven boundedness of $\hat{\boldsymbol{S}}(t)$ and $\hat{\boldsymbol{R}}(t)$ implies that their filtered versions defined in (22) are bounded for all $t \geq 0$ due to the BIBO property of (23). Moreover, the position estimate $\hat{\boldsymbol{x}}(t)$ is guaranteed to belong to the compact region:

$$
\hat{\boldsymbol{x}}(t) \in\left[\inf _{t_{a} \leq \tau \leq t} \hat{\boldsymbol{R}}_{f}(t)^{-1} \hat{\boldsymbol{S}}_{f}(t), \sup _{t_{a} \leq \tau \leq t} \hat{\boldsymbol{R}}_{f}(t)^{-1} \hat{\boldsymbol{S}}_{f}(t)\right],
$$

for all $t \geq t_{a}$, where $t_{a}$ denotes the activation time, i.e. the first time instant that all singular values of $\hat{\boldsymbol{R}}_{f}(t)$ exceed the singularity threshold $\bar{\theta}$.

Therefore, the estimation error $\tilde{\boldsymbol{x}}(t)=\hat{\boldsymbol{x}}(t)-\boldsymbol{x}$ is bounded with respect to bounded disturbances $\eta_{d}(t)$ and $\boldsymbol{\eta}_{y}(t), \forall t \geq t_{a}$.

\section{B. Analysis of the bias introduced by the time-discretization of the algorithm}

In the following, the asymptotic bias on the estimates due to time-discretization will be characterized by considering the discrete counterpart of (18) for $t \rightarrow \infty$ (i.e., when (18) tends to a LTI dynamical system). The discrete-time auxiliary signals can be considered produced by the linear filters:

$$
\begin{aligned}
\boldsymbol{r}_{y}(k) & \triangleq F(z) \boldsymbol{y}(k), \\
r_{s_{d}}(k) & \triangleq F(z) s_{d}(k), \\
r_{s_{y}}(k) & \triangleq F(z) s_{y}(k),
\end{aligned}
$$

where the transfer function $F(z)$ is given by

$$
F(z)=F_{1}(z)+F_{2}(z)
$$

and where $F_{1}(z)$ and $F_{2}(z)$ are obtained by a backward Euler discretization approach:

$$
\begin{aligned}
F_{i}(z) & =\left.\frac{E_{i}}{s-G_{i}}\right|_{s=(z-1) /(T z)}=\frac{\frac{E_{i} T}{\left(1-G_{i} T\right)} z}{z-\frac{1}{1-G_{i} T}} \\
& =\frac{z \bar{E}_{i}}{z-\bar{G}_{i}}, i=1,2,
\end{aligned}
$$


where $G_{i}=-i \omega, E_{i}=\lim _{t \rightarrow \infty} K_{i}(t, t), \bar{E}_{i}=\frac{E_{i} T}{\left(1-G_{i} T\right)}$ and $\bar{G}_{i}=\frac{1}{1-G_{i} T}$.

We assume that the measurement noises are additive on $y(k)$ and $d(k)$.

$$
\begin{aligned}
& \hat{d}(k)=d(k)+v(k), \\
& \hat{\boldsymbol{y}}(k)=\boldsymbol{y}(k)+\boldsymbol{w}(t),
\end{aligned}
$$

where $v(t) \in \mathbb{R}$ and $\boldsymbol{w}(\boldsymbol{t}) \in \mathbb{R}^{3}$ are zero-mean white noises with respective variances $\sigma_{v}^{2}$ and $\boldsymbol{\sigma}_{w}^{2}=\left[\sigma_{w 1}^{2}, \sigma_{w 2}^{2}, \sigma_{w 3}^{2}\right]^{\top}$. Thus, by defining

$$
\begin{aligned}
& \hat{s}_{d}(k) \triangleq d(k)^{2}+2 d(k) v(k)+v(k)^{2}, \\
& \hat{\boldsymbol{s}}_{y}(k) \triangleq \boldsymbol{y}(k)^{\top} \boldsymbol{y}(k)+2 \boldsymbol{y}(k)^{\top} \boldsymbol{w}(k)+\boldsymbol{w}(k)^{\top} \boldsymbol{w}(k),
\end{aligned}
$$

the auxiliary signals produced by the discretized filter become:

$$
\begin{aligned}
\hat{r}_{s_{d}}(k) & =F(z) \cdot \hat{s}_{d}(k)=r_{s_{d}}(k)+e_{2 d v}(k)+e_{v^{2}}(k), \\
\hat{r}_{s_{y}}(k) & =F(z) \cdot \hat{s}_{y}(k)=r_{s_{y}}(k)+e_{2 y w}(k)+e_{w w}(k), \\
\hat{\boldsymbol{r}}_{y}(k) & =F(z) \cdot \hat{\boldsymbol{y}}(k)=\boldsymbol{r}_{y}(k)+\boldsymbol{e}_{w}(k),
\end{aligned}
$$

with

$$
\begin{aligned}
e_{2 d v}(k) & =F(z) \cdot 2 d(k) v(k), \\
e_{v^{2}}(k) & =F(z) \cdot v(k)^{2}, \\
e_{2 y w}(k) & =F(z) \cdot 2 y(k)^{\top} w(k), \\
e_{w w}(k) & =F(z) \cdot \boldsymbol{w}(k)^{\top} \boldsymbol{w}(k), \\
\boldsymbol{e}_{w}(k) & =F(z) \cdot \boldsymbol{w}(k) .
\end{aligned}
$$

The location of the source is estimated based on the equation

$$
\hat{r}_{s_{d}}(k)-\hat{r}_{s_{y}}(k)=-2 \hat{\boldsymbol{r}}_{y}(k)^{\top} \hat{\boldsymbol{x}} .
$$

Replacing the expressions in (38) with the terms in (37) and left-multiplying by $\boldsymbol{r}_{y}(k)$, we obtain

$$
\begin{gathered}
\boldsymbol{r}_{y}(k) r_{s_{d}}(k)+\boldsymbol{r}_{y}(k) e_{2 d v}(k)+\boldsymbol{r}_{y}(k) e_{v^{2}}(k) \\
-\boldsymbol{r}_{y}(k) r_{s_{y}}(k)-\boldsymbol{r}_{y}(k) e_{2 y w}(k)-\boldsymbol{r}_{y}(k) e_{w w}(k) \\
\quad=-2\left[\boldsymbol{r}_{y}(k) \boldsymbol{r}_{y}(k)^{\top}+\boldsymbol{r}_{y}(k) \boldsymbol{e}_{w}(k)^{\top}\right] \hat{\boldsymbol{x}}
\end{gathered}
$$

Applying the $\mathbb{E}[\cdot]$ expectation operator to both sides of (39), after some algebra, we get

$$
\begin{gathered}
\boldsymbol{R}_{r_{y} r_{s_{d}}}+\boldsymbol{R}_{r_{y} e_{2 d v}}+\boldsymbol{R}_{r_{y} e_{v^{2}}}-\boldsymbol{R}_{r_{y} r_{s y}} \\
\quad-\boldsymbol{R}_{r_{y} e_{2 y w}}-\boldsymbol{R}_{r_{y} e_{w w}}=-2 \boldsymbol{R}_{r_{y}} \hat{\boldsymbol{x}},
\end{gathered}
$$

where $\boldsymbol{R}_{a b} \triangleq \mathbb{E}[a b]$ and $\boldsymbol{R}_{a} \triangleq \mathbb{E}\left[a a^{\top}\right]$. Comparing (40) to the following equation, that expresses the relation between covariances in the absence of the measurement noise

$$
\boldsymbol{R}_{r_{y} r_{s_{d}}}-\boldsymbol{R}_{r_{y} r_{s_{y}}}=-2 \boldsymbol{R}_{r_{y}} \boldsymbol{x},
$$

it is straightforward to obtain the estimation bias

$$
\begin{aligned}
& \mathbb{E}[\Delta \boldsymbol{x}]=\mathbb{E}[\hat{\boldsymbol{x}}-\boldsymbol{x}] \\
& \quad=-\left(2 \boldsymbol{R}_{r_{y}}\right)^{-1}\left[\boldsymbol{R}_{r_{y} r_{s_{d}}}+\boldsymbol{R}_{r_{y} e_{v^{2}}}-\boldsymbol{R}_{r_{y} r_{s_{y}}}-\boldsymbol{R}_{r_{y} e_{w w}}\right],
\end{aligned}
$$

where

$$
\begin{aligned}
& \boldsymbol{R}_{r_{y} e_{2 d v}}=0, \\
& \boldsymbol{R}_{r_{y} e_{2 y w}}=0, \\
& \boldsymbol{R}_{r_{y} e_{v^{2}}}=\left[\frac{\bar{E}_{1}}{\left(1-\bar{G}_{1}\right)}+\frac{\bar{E}_{2}}{\left(1-\bar{G}_{2}\right)}\right]^{2} \overline{\boldsymbol{y}} \sigma_{v}^{2}, \\
& \boldsymbol{R}_{r_{y} e_{w w}}=\left[\frac{\bar{E}_{1}}{\left(1-\bar{G}_{1}\right)}+\frac{\bar{E}_{2}}{\left(1-\bar{G}_{2}\right)}\right]^{2} \overline{\boldsymbol{y}} \mathbb{E}\left[\boldsymbol{w}^{\top} \boldsymbol{w}\right],
\end{aligned}
$$

with $\mathbb{E}\left[\boldsymbol{w}^{\top} \boldsymbol{w}\right]=\left(\sigma_{w 1}^{2}+\sigma_{w 2}^{2}+\sigma_{w 3}^{2}\right)$, and $\overline{\boldsymbol{y}}$ denoting the expected value of $\boldsymbol{y}(k)$. See the Appendix for the proof of (42).

\section{DRIFTING TARGET}

The analysis in the previous sections is based on the constraint (7), which assumes that the target is stationary with respect to the reference frame that encodes the known trajectory $\boldsymbol{y}(t)$ of the agent, i.e. $\boldsymbol{x}$ is a constant vector. From now on we are trying to exploit the possibility of relaxing the stationary constraint by assuming a persistent drift on the source. All the aforementioned conditions remain unchanged except the motion of the source, which subsumes the following assumption.

Assumption 3: The target trajectory $\boldsymbol{x}(t)$ is differentiable and there exist $M_{3}>0$ and $\epsilon>0$, such that for all $t \geq 0$,

$$
\begin{aligned}
\|\boldsymbol{x}(t)\| & \leq M_{3}, \\
\|\dot{\boldsymbol{x}}(t)\| & \leq \epsilon .
\end{aligned}
$$

(43) implies that the drift is within a bounded range which avoids collinear measurements. Moreover, the velocity of the drift is also assumed to be bounded.

In this regard, the counterpart of constraint (4) which governs the drifting source localization problem becomes:

$$
\frac{d}{d t}\left\{d^{2}(t)\right\}=2 \frac{d}{d t} \boldsymbol{y}(t)^{\top}(\boldsymbol{y}(t)-\boldsymbol{x}(t))-2 \frac{d}{d t} \boldsymbol{x}(t)^{\top}(\boldsymbol{y}(t)-\boldsymbol{x}(t)) .
$$

The following intermediate result will be instrumental to characterize the robustness of the method in facing a persistent drift.

Lemma 6.1: Under the Assumptions 1 and 3, there exist a $k_{1}>0$ depending on $M_{1}, M_{2}$ (defined in Assumption 1), $M_{3}$ and $\omega$ (tuning parameter of the kernel), verifying:

$$
\left|\left[V_{K} s_{d}^{(1)}\right](t)-\left[V_{K} s_{y}^{(1)}\right](t)+2\left[\boldsymbol{V}_{\boldsymbol{K}} \boldsymbol{y}^{(\mathbf{1})}\right](t)^{\top} \boldsymbol{x}(t)\right| \leq k_{1} \epsilon .
$$

Proof: By exploiting the notations defined in (5), we can rewrite (45) as

$$
s_{d}^{(1)}(t)=s_{y}^{(1)}(t)-2 \boldsymbol{y}^{(1)}(t)^{\top} \boldsymbol{x}(t)-2 \boldsymbol{x}^{(1)}(t)^{\top}[\boldsymbol{y}(t)-\boldsymbol{x}(t)] .
$$

Applying the Volterra operator to both side of (46), one can obtain

$$
\begin{array}{r}
{\left[V_{K} s_{d}^{(1)}\right](t)=\left[V_{K} s_{y}^{(1)}\right](t)-2\left[\boldsymbol{V}_{\boldsymbol{K}} \boldsymbol{y}^{(\mathbf{1})^{\top}} \boldsymbol{x}\right](t)} \\
-2\left[\boldsymbol{V}_{\boldsymbol{K}} \boldsymbol{x}^{(\mathbf{1})^{\top}}(\boldsymbol{y}-\boldsymbol{x})\right](t),
\end{array}
$$


where

$$
\begin{aligned}
{\left[\boldsymbol{V}_{\boldsymbol{K}} \boldsymbol{x}^{(\mathbf{1})^{\top}}(\boldsymbol{y}-\boldsymbol{x})\right](t) } & =\int_{0}^{t} K(t, \tau) \boldsymbol{x}^{(\mathbf{1})}(\tau)^{\top}[\boldsymbol{y}(\tau)-\boldsymbol{x}(\tau)] d \tau \\
& \leq \int_{0}^{t} K(t, \tau) d \tau\left(M_{1}+M_{3}\right) \epsilon \\
& =\left[P_{1}(t)+P_{2}(t)\right]\left(M_{1}+M_{3}\right) \epsilon,
\end{aligned}
$$

and

$$
\begin{aligned}
& P_{1}(t)=e^{-2 \omega t}\left(\frac{e^{\omega t}-1}{\omega}-\frac{e^{2 \omega t}-1}{2 \omega}\right), \\
& P_{2}(t)=e^{-\omega t}\left(\frac{e^{\omega t}-1}{\omega}-t\right) .
\end{aligned}
$$

Moreover,

$$
\begin{aligned}
{\left[V_{K} y^{(1)^{\top}} x\right](t)=} & \int_{0}^{t} K(t, \tau) \boldsymbol{y}^{(1)}(\tau)^{\top} \boldsymbol{x}(\tau) d \tau \\
= & e^{-2 \omega t} \int_{0}^{t} e^{\omega \tau} \boldsymbol{y}^{(1)}(\tau)^{\top} \boldsymbol{x}(\tau) d \tau \\
& -e^{-2 \omega t} \int_{0}^{t} e^{2 \omega \tau} \boldsymbol{y}^{(1)}(\tau)^{\top} \boldsymbol{x}(\tau) d \tau \\
& +e^{-\omega t} \int_{0}^{t} e^{\omega \tau} \boldsymbol{y}^{(1)}(\tau)^{\top} \boldsymbol{x}(\tau) d \tau \\
& -e^{-\omega t} \int_{0}^{t} \boldsymbol{y}^{(1)}(\tau)^{\top} \boldsymbol{x}(\tau) d \tau .
\end{aligned}
$$

In view of the rule of integration by parts, (49) writes

$$
\begin{aligned}
{\left[V_{K} y^{(1)^{\top}} x\right](t)=} & e^{-2 \omega t}\left[\left(\int_{0}^{\tau} e^{\omega s} \boldsymbol{y}^{(1)}(s) d s\right)^{\top} \boldsymbol{x}(\tau)\right]_{0}^{t} \\
& -e^{-2 \omega t}\left[\left(\int_{0}^{\tau} e^{2 \omega s} \boldsymbol{y}^{(1)}(s) d s\right)^{\top} \boldsymbol{x}(\tau)\right]_{0}^{t} \\
& +e^{-\omega t}\left[\left(\int_{0}^{\tau} e^{\omega s} \boldsymbol{y}^{(1)}(s) d s\right)^{\top} \boldsymbol{x}(\tau)\right]_{0}^{t} \\
& -e^{-\omega t}\left[\left(\int_{0}^{\tau} \boldsymbol{y}^{(1)}(s) d s\right)^{\top} \boldsymbol{x}(\tau)\right]_{0}^{t}-f(t) \\
= & {\left[\boldsymbol{V}_{\boldsymbol{K}} \boldsymbol{y}^{(\mathbf{1})}\right](t)^{\top} \boldsymbol{x}(t)-f(t), }
\end{aligned}
$$

where

$$
\begin{aligned}
f(t)= & e^{-2 \omega t} \int_{0}^{t}\left(\int_{0}^{\tau} e^{\omega s} \boldsymbol{y}^{(1)}(s) d s\right)^{\top} \boldsymbol{x}^{(1)}(\tau) d \tau \\
& -e^{-2 \omega t} \int_{0}^{t}\left(\int_{0}^{\tau} e^{2 \omega s} \boldsymbol{y}^{(1)}(s) d s\right)^{\top} \boldsymbol{x}^{(1)}(\tau) d \tau \\
& +e^{-\omega t} \int_{0}^{t}\left(\int_{0}^{\tau} e^{\omega s} \boldsymbol{y}^{(1)}(s) d s\right)^{\top} \boldsymbol{x}^{(1)}(\tau) d \tau \\
& -e^{-\omega t} \int_{0}^{t}\left(\int_{0}^{\tau} \boldsymbol{y}^{(1)}(s) d s\right)^{\top} \boldsymbol{x}^{(1)}(\tau) d \tau .
\end{aligned}
$$

Moreover

$$
\begin{aligned}
|f(t)| \leq & \left|e^{-2 \omega t}\left[\frac{\left(e^{\omega t}-1\right)}{\omega^{2}}+\frac{t}{\omega}\right]\right| M_{2} \epsilon \\
& +\left|-e^{-2 \omega t}\left[\frac{\left(e^{2 \omega t}-1\right)}{4 \omega^{2}}+\frac{t}{2 \omega}\right]\right| M_{2} \epsilon \\
& +\left|e^{-\omega t}\left[\frac{\left(e^{\omega t}-1\right)}{\omega^{2}}+\frac{t}{\omega}\right]\right| M_{2} \epsilon \\
& +\left|-e^{-\omega t}\left(\frac{t^{2}}{2}\right)\right| M_{2} \epsilon .
\end{aligned}
$$

Thus, there exists a $k_{1}>0$ satisfying

$$
\begin{aligned}
& \left|\left[V_{K} s_{d}^{(1)}\right](t)-\left[V_{K} s_{y}^{(1)}\right](t)+2\left[\boldsymbol{V}_{\boldsymbol{K}} \boldsymbol{y}^{(\mathbf{1})}\right](t)^{\top} \boldsymbol{x}(t)\right| \\
& =\left|-2\left[\boldsymbol{V}_{\boldsymbol{K}} \boldsymbol{x}^{(\mathbf{1})^{\top}}(\boldsymbol{y}-\boldsymbol{x})\right](t)+2 f(t)\right| \leq k_{1} \epsilon .
\end{aligned}
$$

According to the Lemma 6.1, we have the following result:

Theorem 6.1: Under Assumptions 1 and 3, there exists a $k_{2}>0$ determined by $M_{1}, M_{2}, M_{3}, \omega$ and $g$ (the time constant of covariance filter), such that the localization error subsumes the asymptotic bound $\lim _{t \rightarrow \infty} \sup \|\boldsymbol{x}(t)-\hat{\boldsymbol{x}}(t)\|=k_{2} \epsilon$.

Proof: By applying covariance filtering to (47), we obtain the augmented vector equation:

$$
\left[\boldsymbol{V}_{\boldsymbol{G}} \boldsymbol{P}\right](t)+\left[\boldsymbol{V}_{\boldsymbol{G}} \boldsymbol{Q}\right](t)=\left[\boldsymbol{V}_{\boldsymbol{G}} \boldsymbol{T} \boldsymbol{x}\right](t),
$$

where

$$
\begin{aligned}
{\left[\boldsymbol{V}_{\boldsymbol{G}} \boldsymbol{P}\right](t) } & =\int_{0}^{t} e^{-g(t-\tau)} \boldsymbol{P}(\tau) d \tau=\boldsymbol{S}_{f}(t), \\
\boldsymbol{P}(t) & =\left[\boldsymbol{V}_{\boldsymbol{K}} \boldsymbol{y}^{(\mathbf{1})}\right](t) \frac{\left[V_{K} s_{d}^{(1)}\right](t)-\left[V_{K} s_{y}^{(1)}\right](t)}{-2}=\boldsymbol{S}(t), \\
{\left[\boldsymbol{V}_{\boldsymbol{G}} \boldsymbol{Q}\right](t) } & =\int_{0}^{t} e^{-g(t-\tau)} \boldsymbol{Q}(\tau) d \tau, \\
\boldsymbol{Q}(t) & =\left[\boldsymbol{V}_{\boldsymbol{K}} \boldsymbol{y}^{(\mathbf{1})}\right](t)\left(-\left[\boldsymbol{V}_{\boldsymbol{K}} \boldsymbol{x}^{(\mathbf{1}) \top}(\boldsymbol{y}-\boldsymbol{x})\right](t)+f(t)\right), \\
{\left[\boldsymbol{V}_{\boldsymbol{G}} \boldsymbol{T} \boldsymbol{x}\right](t) } & =\int_{0}^{t} e^{-g(t-\tau)} \boldsymbol{T}(\tau) \boldsymbol{x}(\tau) d \tau=\boldsymbol{R}_{f}(t), \\
\boldsymbol{T}(t) & =\left[\boldsymbol{V}_{\boldsymbol{K}} \boldsymbol{y}^{(\mathbf{1})}\right](t)\left[\boldsymbol{V}_{\boldsymbol{K}} \boldsymbol{y}^{(\mathbf{1})}\right](t)^{\top}=\boldsymbol{R}(t) .
\end{aligned}
$$

By the same line of reasoning, from (49) and (50), we have

$$
\left[\boldsymbol{V}_{\boldsymbol{G}} \boldsymbol{T} \boldsymbol{x}\right](t)=\left[\boldsymbol{V}_{\boldsymbol{G}} \boldsymbol{R}\right](t) \boldsymbol{x}(t)-\boldsymbol{T}_{e}(t) .
$$

Moreover, there exist a $\boldsymbol{k}_{3} \in \mathbb{R}_{>0}^{3}$ such that $\left|\boldsymbol{T}_{e}(t)\right| \leq \boldsymbol{k}_{3} \epsilon$.

Therefore, the estimation error caused by the drift is

$$
\|\tilde{\boldsymbol{x}}(t)\|=\|\boldsymbol{x}(t)-\hat{\boldsymbol{x}}(t)\|=\left\|\boldsymbol{R}_{f}(t)^{-1}\left(\left[\boldsymbol{V}_{\boldsymbol{G}} \boldsymbol{Q}\right](t)+\boldsymbol{T}_{e}(t)\right)\right\| .
$$

In the light of the BIBO-stability and the linearity properties of the Volterra kernel $V_{G}$ in covariance filtering, and thanks to (48) and (50), under the P.E. condition (24), the estimation error of the proposed algorithm for tracking drift is bounded and, meanwhile, its upper bound is proportional to the upper bound of the drift rate, i.e. there exist a $k_{2}>0$ obtained from $M_{1}, M_{2}, M_{3}, \omega$ and $g$, such that for time $t \rightarrow \infty$, $\sup \|\tilde{\boldsymbol{x}}(t)\|=\sup \|\boldsymbol{x}(t)-\hat{\boldsymbol{x}}(t)\|=k_{2} \epsilon$.

Theorem 6.1 indicates that the proposed algorithm is able to track the location of a drifting source with tunable accuracy. 


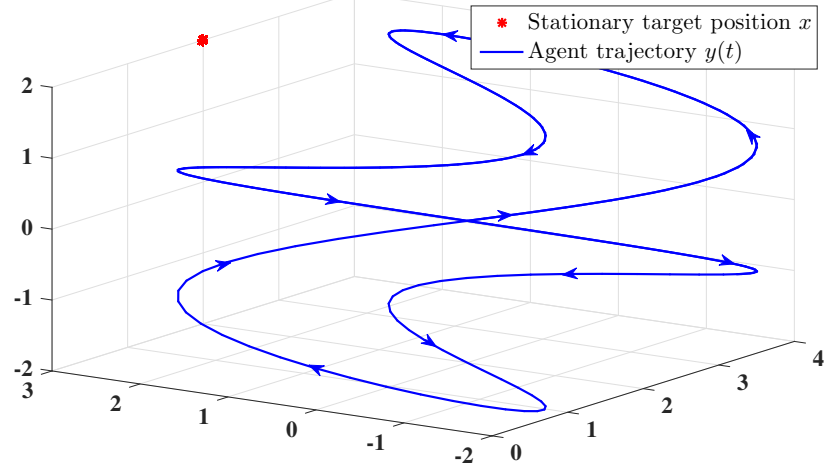

Fig. 1: Positional relationship of the stationary target and the agent trajectory considered in Example VII-A (stationary target in noise-free conditions).

\section{Simulation RESUlts AND COMPARISONS}

In this section, the proposed method is compared with a continuous-time recursive least-square-based (CT-RLS) adaptive estimator presented in [17] and a discrete-time recursive (DT-R) method [18], which are range-based single-agent localization algorithms with fading memory mechanism characterized by remarkable immunity to measurement noise and drifts. The simulation is carried out in Matlab/Simulation environment, with a sampling time $T_{s}=10^{-3} s$.

The algorithm is simulated for localizing both a stationary source position $\boldsymbol{x}=[2,3,2]^{\top}(m)$ and a drifting source $\boldsymbol{x}(t)=[2+\sin 0.01 t, 3+\cos 0.01 t, 2]^{\top}(m)$ in four different scenarios taking the measurement noise into consideration. For all $t \geq 0$, the agent moves along the trajectory $\boldsymbol{y}(t)=$ $\left[2+2 \sin t, 2 \sin \left(2 t+\frac{\pi}{2}\right), 2 \sin 0.5 t\right]^{\top}(m)$. We set the tuning parameters of the kernel-based algorithm as $\omega=1, g=1$ and the threshold $\bar{\theta}=10^{-15}$, while the other two estimators are tuned by $\alpha=1, \beta=1, \boldsymbol{P}(0)=10^{6} \cdot \boldsymbol{I}_{3 \times 3}$, all other initial values involved are set to zero for [17] and $\rho_{l}^{2}=0.5$, $\beta=0.99, T_{s}=0.5 s$ and $\epsilon=10^{-6}$ for [18].

\section{A. Stationary target: estimation under noise-free condition}

The positional relationship of the stationary $\operatorname{target} \boldsymbol{x}$ and the trajectory of the agent $\boldsymbol{y}(t)$ are described by Fig. 1 .

In Fig. 2, the results are shown in the noise-free scenario. Activated at around $t=0.2 s$, the kernel-based estimator shows a very fast convergence rate and high accuracy.

\section{B. Stationary target: estimation in noisy scenario}

In this example, an additive measurement noise simulated as a uniformly distributed random signal within the range of $[-0.5,0.5]$ affects the distance $d(t)$ with $S N R=23.36$, as depicted in Fig. 3.

As reported by Fig. 4, in spite of the high-rate convergence, the proposed method also shows absolute advantages in terms of robustness against measurement noise. In steady state, 10001 samples of the estimates within the time interval

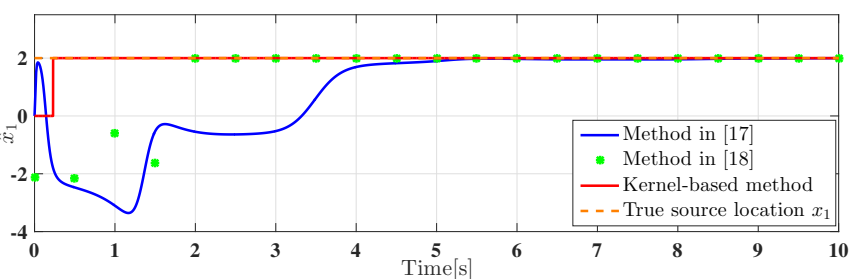

(a)

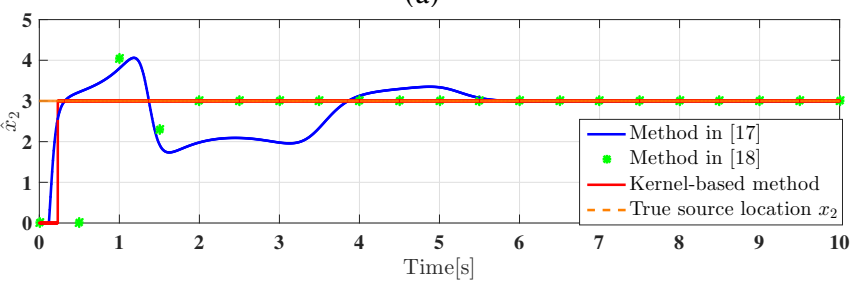

(b)

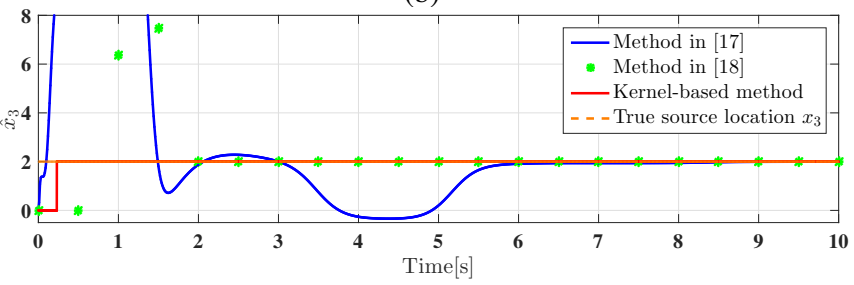

(c)

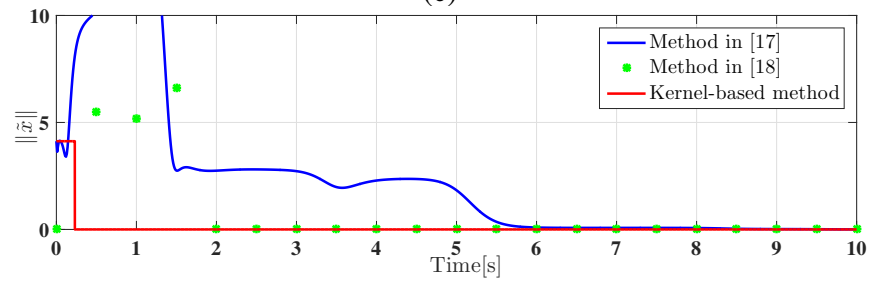

(d)

Fig. 2: (a)-(c): Time behavior of the two methods in estimating a stationary target location $\boldsymbol{x}=[2,3,2]^{\top}(m)$ with the agent location $\boldsymbol{y}(t)=\left[2+2 \sin (t), 2 \sin \left(2 t+\frac{\pi}{2}\right), 2 \sin (0.5 t)\right]^{\top}(m)$ under a noise-free condition. (d): Norms of the estimation errors.

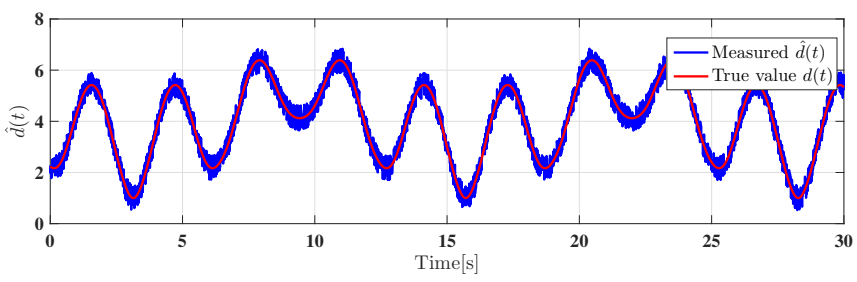

Fig. 3: Measured distance signal $\hat{d}(t)$ and pure signal $d(t)$ with stationary target

$t \in[20,30]$ are taken to calculate the Root Mean Square Error (RMSE) defined as

$$
R M S E=\sqrt{\frac{\sum_{i=20001}^{30001}\|\tilde{\boldsymbol{x}}(i)\|^{2}}{10001}} .
$$




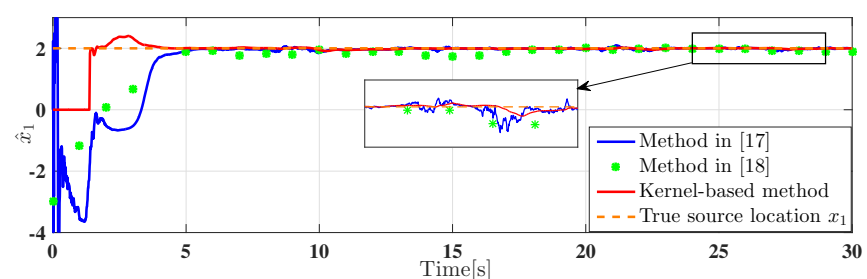

(a)

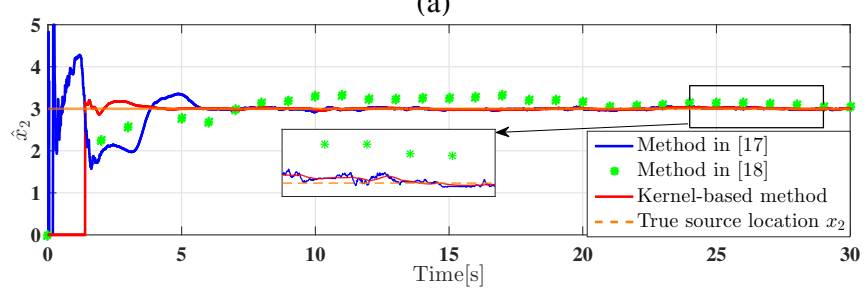

(b)

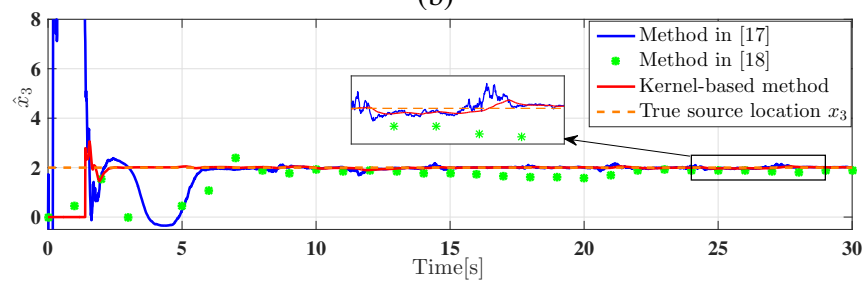

(c)

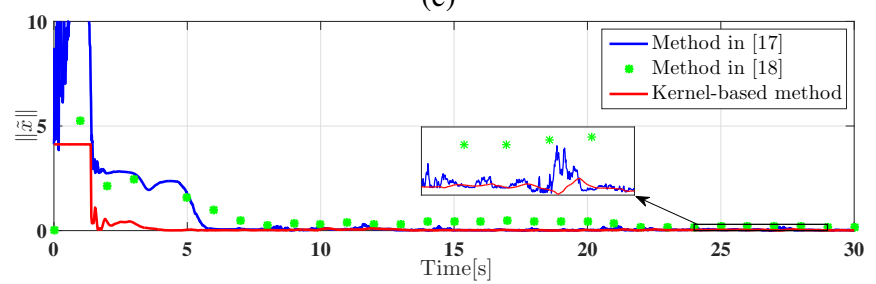

(d)

Fig. 4: (a)-(c): Time behavior of the two methods in estimating a stationary source location $\boldsymbol{x}=[2,3,2]^{\top}(m)$ with the agent location $\boldsymbol{y}(t)=\left[2+2 \sin (t), 2 \sin \left(2 t+\frac{\pi}{2}\right), 2 \sin (0.5 t)\right]^{\top}(m)$ under a uniformly distributed measurement noise ranging from $[-0.5,0.5]$. (d): Norms of the estimation errors.

and variance of the estimation error (see Table I), which intuitively illustrate the noise immunity of the three position estimators.

\section{Drifting target: estimation under noise-free condition}

In this case, a source which has small drift around the original position $[2,3,2]^{\top}$, namely $\forall t \geq 0$, $\boldsymbol{x}(t)=[2+\sin 0.01 t, 3+\cos 0.01 t, 2]^{\top}(m)$, is considered. As shown in the 3-D map of the trajectories in Fig. 5, the drift is added on the first two elements of $x(t)$ with a relatively small rate with an upper-bound of $\frac{\sqrt{2}}{100}$. On the other hand, the drift is bounded, which verifies (43). Applying the algorithms with the same parameters, we can obtain the tracking results shown in Fig. 6. Notice that in [18], the problem of localizing a persistent drifting source is not addressed, therefore, only

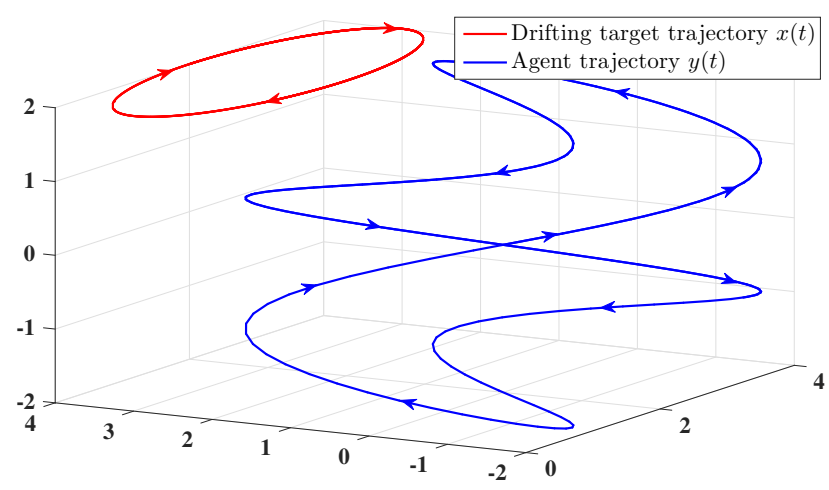

Fig. 5: Positional relationship of the drifting target and the agent trajectory considered in Example VII-C (drifting target in noise-free conditions).

the performance of the CT-RLS method in [17] will be compared with.

According to the Fig. 5-6, the kernel-based estimation, activated at around $1.4 \mathrm{~s}$, performs a fast and smooth tracking of the drifting source location. The estimation error is bounded and fluctuates around $0.05 \mathrm{~m}$ after reaching the steady state according to Fig. 6d.

\section{Drifting target: estimation in noisy scenario}

In this scenario we analyze the behavior of the estimator in presence of a uniformly distributed noise signal which adds to the distance measurements $\hat{d}(t)(S N R=24.36)$. The measured noisy distance signal is shown in Fig. 7 and the corresponding estimation results are shown in Fig. 8.

Compared to the noise-free scenario, the algorithm takes longer time (around $3 s$ in this case) in the initial phase to track the drifting source. Also in this final simulation run, the kernelbased method has shown a superior immunity to measurement noise, and the estimation error reaches a steady level with the value of approximately $0.05 \mathrm{~m}$. Similarly with example VII-B, the steady state RMSEs and variances of the estimation error $(t \in[150,200] \mathrm{s}, 50001$ points are taken) are listed in Table II.

\section{CONCLUDING REMARKS}

In this paper, a single-agent range-based localization algorithm arising from the algebra of Volterra operators has been proposed. The advantage of the devised algorithm is that the source location can be determined in finite-time. The kernelbased methodology is capable to track also a drifting target with a bounded estimation error. Simulation results show a good immunity with respect to bounded measurement noise.

Further research efforts will be made to optimize the choice of the tuning parameters trading off the convergence rate, the estimation error and the bias caused by discretization. Moreover, another direction of research will be on adaptive laws specific for the on-line localization of a mobile source. 
TABLE I: RMSE and variance of three localization algorithms Example VII-B

\begin{tabular}{|c|c|c|c|}
\hline Methods & Kernel-based method & CT-RLS method [17] & DT-R method [18] \\
\hline RMSE & 0.0310 & 0.0553 & 0.2381 \\
\hline$\sigma^{2}$ & $1.35 \times 10^{-4}$ & $1.10 \times 10^{-3}$ & $8.80 \times 10^{-3}$ \\
\hline
\end{tabular}

TABLE II: RMSE and variance of two localization algorithms in Example VII-D

\begin{tabular}{|c|c|c|}
\hline Methods & Kernel-based method & CT-RLS method [17] \\
\hline RMSE & 0.0600 & 0.0723 \\
\hline$\sigma^{2}$ & $3.93 \times 10^{-4}$ & $1.20 \times 10^{-3}$ \\
\hline
\end{tabular}

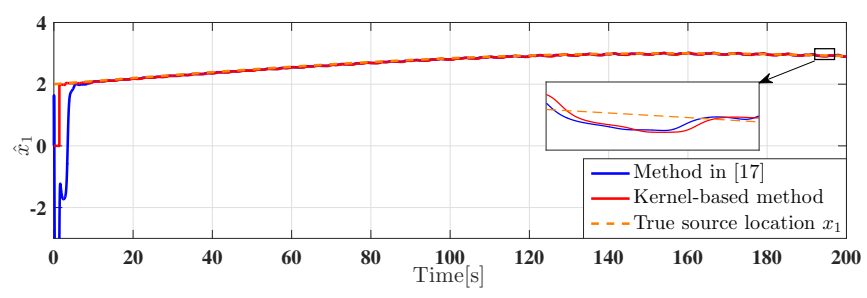

(a)

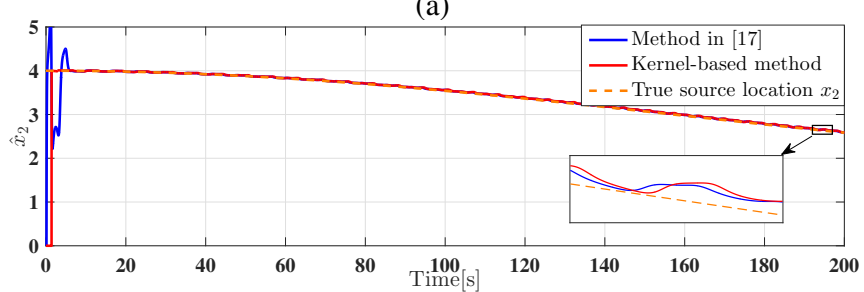

(b)

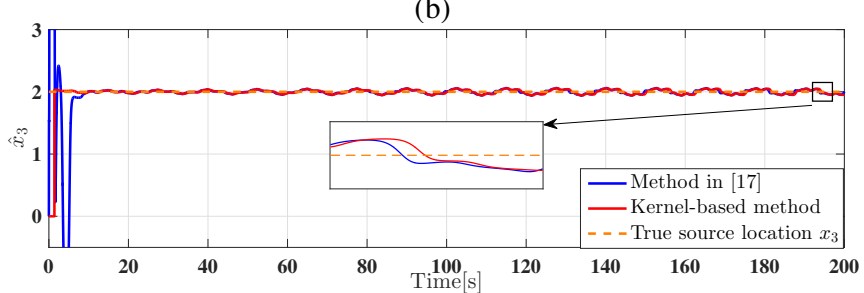

(c)

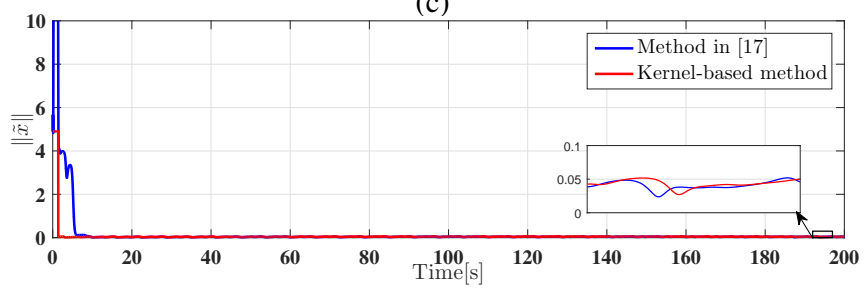

(d)

Fig. 6: (a)-(c): Time behavior of the two methods in estimating three elements of a drifting source location $\boldsymbol{x}(t)=$ $[2+\sin 0.01 t, 3+\cos 0.01 t, 2]^{\top}(m)$ with the agent location $\boldsymbol{y}(t)=\left[2+2 \sin (t), 2 \sin \left(2 t+\frac{\pi}{2}\right), 2 \sin (0.5 t)\right]^{\top}(m)$ under a no-noise condition. (d): Norms of the estimation errors.

$$
\begin{aligned}
& \text { APPENDIX } \\
& \text { PROOF OF (42) } \\
& \boldsymbol{R}_{r_{y} e_{2 d v}}=\mathbb{E}\left[\boldsymbol{r}_{y} \cdot e_{2 d v}\right] \\
&=\mathbb{E}\left[\sum_{i=1}^{2} \sum_{j=1}^{2} \boldsymbol{r}_{y, i}(k) e_{2 d v, j}(k)\right],
\end{aligned}
$$

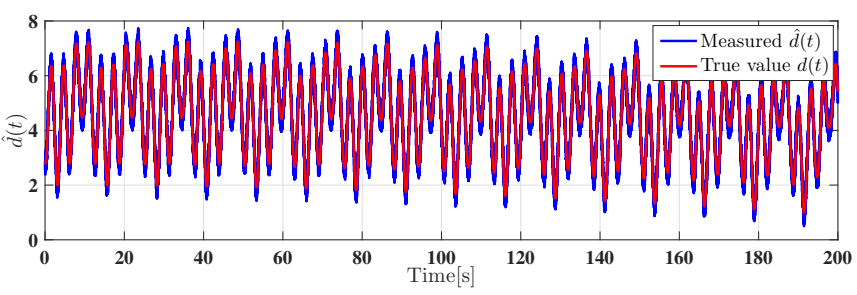

Fig. 7: Noisy distance signal $\hat{d}(t)$ and pure signal $d(t)$ in drifting source case.

where $\boldsymbol{r}_{y, i}(k)=F_{i}(z) \cdot \boldsymbol{y}(k)$ and $e_{2 d v, j}(k)=F_{j}(z) \cdot e_{2 d v}(k)$ with $j=1,2$ or equivalently,

$$
\begin{aligned}
& \boldsymbol{r}_{y, i}(k)=\bar{G}_{i} \boldsymbol{r}_{y, i}(k-1)+\bar{E}_{i} \boldsymbol{y}(k), \\
& e_{2 d v, i}(k)=\bar{G}_{i} e_{2 d v, i}(k-1)+\bar{E}_{i}[2 d(k) v(k)] .
\end{aligned}
$$

Therefore,

$$
\begin{aligned}
& \mathbb{E}\left[\boldsymbol{r}_{y, 1}(k) e_{2 d v, 1}(k)\right]= \\
& \quad \mathbb{E}\left[\bar{G}_{1}^{2} \boldsymbol{r}_{y, 1}(k-1) e_{2 d v, 1}(k-1)\right]+\mathbb{E}\left[\bar{E}_{1}^{2} \boldsymbol{y}(k) 2 d(k) v(k)\right] \\
& \quad+\mathbb{E}\left[\bar{G}_{1} \bar{E}_{1} \boldsymbol{r}_{y, 1}(k-1) \cdot 2 d(k) v(k)\right] \\
& \quad+\mathbb{E}\left[\bar{G}_{1} \bar{E}_{1} \boldsymbol{y}(k) \cdot e_{2 d v, 1}(k-1)\right]
\end{aligned}
$$

From (54) one can obtain

$$
\left(1-\bar{G}_{1}^{2}\right) \mathbb{E}\left[\boldsymbol{r}_{y, 1}(k) e_{2 d v, 1}(k)\right]=\bar{G}_{1} \bar{E}_{1} \mathbb{E}[\boldsymbol{y}(k)] \mathbb{E}\left[e_{2 d v, 1}(k-1)\right]
$$

Notice that, due to (53), $\mathbb{E}\left[e_{2 d v, 1}(k-1)\right]=0$, thus $\mathbb{E}\left[\boldsymbol{r}_{y, 1}(k) e_{2 d v, 1}(k)\right]=0$. In the same way, one can obtain $\mathbb{E}\left[\boldsymbol{r}_{y, 2}(k) e_{2 d v, 1}(k)\right]=0, \mathbb{E}\left[\boldsymbol{r}_{y, 1}(k) e_{2 d v, 2}(k)\right]=$ $0, \mathbb{E}\left[\boldsymbol{r}_{y, 2}(k) e_{2 d v, 2}(k)\right]=0$, and as a result $\boldsymbol{R}_{r_{y} e_{2 d v}}=0$.

By the same line of reasoning it follows that $\boldsymbol{R}_{r_{y} e_{2 d v}}=0$.

Since that the auxiliary sequence $\boldsymbol{r}_{y}(k)$ is independent with $e_{v^{2}}(k)$ and $e_{\boldsymbol{w} \boldsymbol{w}}(k)$, it also holds that

$$
\begin{aligned}
\boldsymbol{R}_{r_{y} e_{v^{2}}} & =\mathbb{E}\left[\boldsymbol{r}_{y}(k)\right] \cdot \mathbb{E}\left[e_{v^{2}}(k)\right], \\
\boldsymbol{R}_{r_{y} e_{w w}} & =\mathbb{E}\left[\boldsymbol{r}_{y}(k)\right] \cdot \mathbb{E}\left[e_{w w}(k)\right],
\end{aligned}
$$




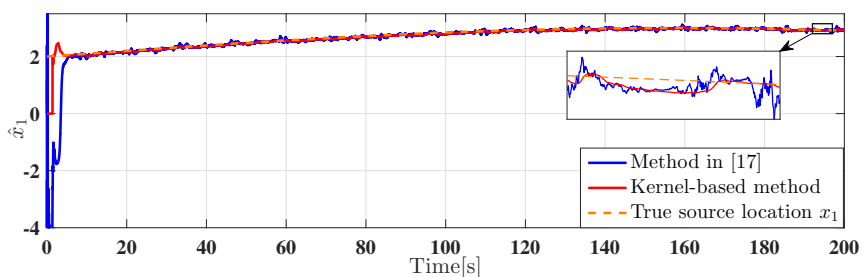

(a)

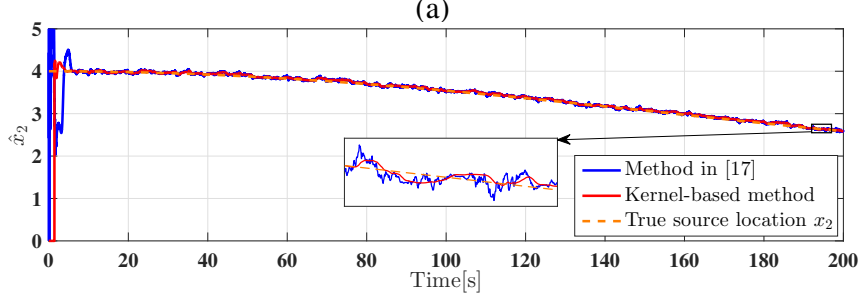

(b)

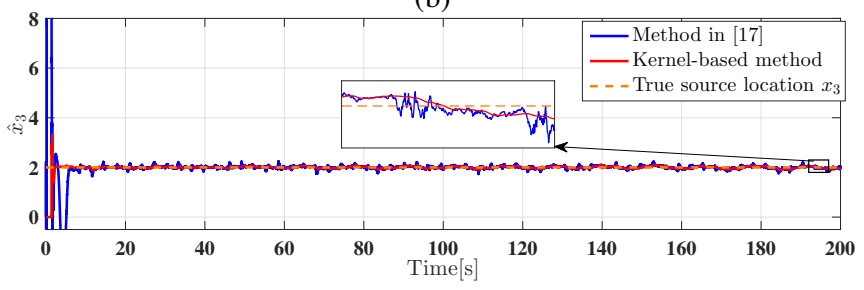

(c)

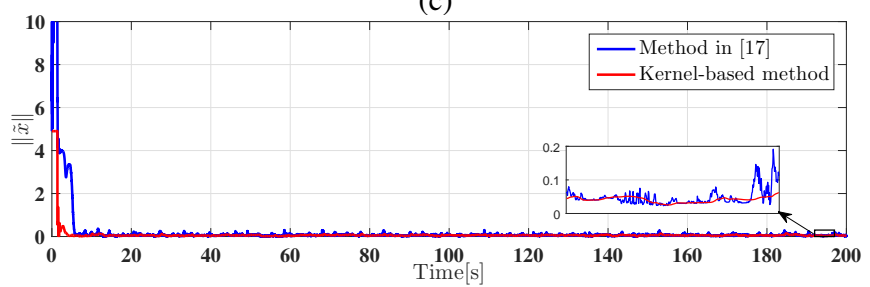

(d)

Fig. 8: (a)-(c): Time behavior of the two methods in estimating three elements of a drifting source location $\boldsymbol{x}(t)=$ $[2+\sin 0.01 t, 3+\cos 0.01 t, 2]^{\top}(m)$ with the agent location $\boldsymbol{y}(t)=\left[2+2 \sin (t), 2 \sin \left(2 t+\frac{\pi}{2}\right), 2 \sin (0.5 t)\right]^{\top}(m)$ under a uniformly distributed measurement noise ranging from $[-0.5,0.5]$. (d): Norms of the estimation errors.

where

$$
\begin{aligned}
\mathbb{E}\left[\boldsymbol{r}_{y}(k)\right] & =\left[\frac{\bar{E}_{1}}{\left(1-\bar{G}_{1}\right)}+\frac{\bar{E}_{2}}{\left(1-\bar{G}_{2}\right)}\right] \overline{\boldsymbol{y}}, \\
\mathbb{E}\left[e_{v^{2}}\right] & =\left[\frac{\bar{E}_{1}}{\left(1-\bar{G}_{1}\right)}+\frac{\bar{E}_{2}}{\left(1-\bar{G}_{2}\right)}\right] \sigma_{v^{2}}, \\
\mathbb{E}\left[e_{w w}\right] & =\left[\frac{\bar{E}_{1}}{\left(1-\bar{G}_{1}\right)}+\frac{\bar{E}_{2}}{\left(1-\bar{G}_{2}\right)}\right] \mathbb{E}\left[\boldsymbol{w}^{\top} \boldsymbol{w}\right],
\end{aligned}
$$

and $\mathbb{E}\left[\boldsymbol{w}^{\top} \boldsymbol{w}\right]=\left(\sigma_{w 1}^{2}+\sigma_{w 2}^{2}+\sigma_{w 3}^{2}\right)$.

\section{ACKNOWLEDGMENT}

The authors would like to thank the Associate Editor and the anonymous Reviewers for the thorough and constructive comments during the review process.

\section{REFERENCES}

[1] G. Indiveri, D. De Palma, and G. Parlangeli, "Single range localization in 3-d: Observability and robustness issues," IEEE Transactions on Control Systems Technology, vol. 24, no. 5, pp. 1853-1860, 2016.

[2] R. Zekavat and R. M. Buehrer, Handbook of position location: Theory, practice and advances. John Wiley \& Sons, 2011, vol. 27.

[3] M. R. Gholami, "Positioning algorithms for wireless sensor networks," PhD Thesis, Chalmers University of Technology, 2011.

[4] J. Wang, R. K. Ghosh, and S. K. Das, "A survey on sensor localization," Journal of Control Theory and Applications, vol. 8, no. 1, pp. 2-11, 2010.

[5] A. Bahr, J. J. Leonard, and M. F. Fallon, "Cooperative localization for autonomous underwater vehicles," The International Journal of Robotics Research, vol. 28, no. 6, pp. 714-728, 2009.

[6] S. E. Webster, R. M. Eustice, H. Singh, and L. L. Whitcomb, "Advances in single-beacon one-way-travel-time acoustic navigation for underwater vehicles," The International Journal of Robotics Research, vol. 31, no. 8, pp. 935-950, 2012.

[7] M. Bayat, N. Crasta, A. P. Aguiar, and A. M. Pascoal, "Rangebased underwater vehicle localization in the presence of unknown ocean currents: Theory and experiments," IEEE Transactions on control systems technology, vol. 24, no. 1, pp. 122-139, 2016.

[8] P. K. Sahoo and I.-S. Hwang, "Collaborative localization algorithms for wireless sensor networks with reduced localization error," Sensors, vol. 11, no. 10, pp. 9989-10 009, 2011.

[9] T. Bian, R. Venkatesan, and C. Li, "Design and evaluation of a new localization scheme for underwater acoustic sensor networks," in Proc. IEEE Global Telecommunications Conference (GLOBECOM). IEEE, 2009, pp. 1-5.

[10] Q. Shi, C. He, H. Chen, and L. Jiang, "Distributed wireless sensor network localization via sequential greedy optimization algorithm," IEEE Transactions on Signal Processing, vol. 58, no. 6, pp. 3328-3340, 2010.

[11] J.-P. Shao and Y.-P. Tian, "Cooperative source localisation of multiagent system based on a cooperative pe condition," Control Theory \& Applications, IET, vol. 9, no. 1, pp. 42-51, 2015.

[12] J. Jouffroy and J. Reger, "An algebraic perspective to single-transponder underwater navigation," in Computer Aided Control System Design, 2006 IEEE International Conference on Control Applications, 2006 IEEE International Symposium on Intelligent Control, 2006 IEEE. IEEE, 2006, pp. 1789-1794.

[13] P. Batista, C. Silvestre, and P. Oliveira, "Optimal position and velocity navigation filters for autonomous vehicles," Automatica, vol. 46, no. 4, pp. 767-774, 2010.

[14] _ "Single range aided navigation and source localization: Observability and filter design," Systems \& Control Letters, vol. 60, no. 8, pp. 665-673, 2011.

[15] S. H. Dandach, B. Fidan, S. Dasgupta, and B. D. Anderson, "A continuous time linear adaptive source localization algorithm, robust to persistent drift," Systems \& Control Letters, vol. 58, no. 1, pp. 7-16, 2009.

[16] G. Chai, Z. Lin, and M. Fu, "Consensus-based cooperative source localization of multi-agent systems," in Proc. 32nd Chinese Control Conference (CCC). IEEE, 2013, pp. 6809-6814.

[17] B. Fidan, A. Çamlıca, and S. Güler, "Least-squares-based adaptive target localization by mobile distance measurement sensors," International Journal of Adaptive Control and Signal Processing, vol. 29, no. 2, pp. 259-271, 2015.

[18] G. Indiveri, P. Pedone, , and M. Cuccovillo, "Fixed target 3d localization based on range data only: A recursive least squares approach," IFAC Proceedings Volumes, vol. 45, no. 5, pp. 140-145, 2012, 3rd IFAC Workshop on Navigation, Guidance and Control of Underwater Vehicles. [Online]. Available: http://www.sciencedirect.com/science/article/pii/S1474667016305936 
[19] G. Pin, A. Assalone, M. Lovera, and T. Parisini, "Non-asymptotic kernel-based parametric estimation of continuous-time linear systems," IEEE Transactions on Automatic Control, vol. 61, no. 2, pp. 360-373, 2016.

[20] G. Pin, M. Lovera, A. Assalone, and T. Parisini, "Kernel-based nonasymptotic state estimation for linear continuous-time systems," in Proc. 2013 American Control Conference (ACC), 2013, pp. 3123-3128.

[21] P. Li, G. Pin, T. Parisini, and G. Fedele, "Deadbeat source localization from range-only measurements: A robust kernel-based approach," in American Control Conference (ACC), 2016. American Automatic Control Council (AACC), 2016, pp. 2729-2734.

[22] T. A. Burton, Volterra integral and differential equations. Elsevier, 2005, vol. 202

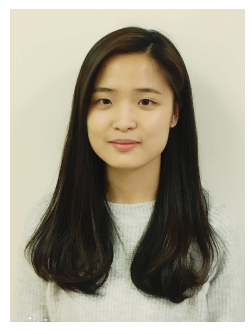

Peng Li received B. Eng degree in Control Technology and Instruments in 2013 from Northeastern University, China. In 2014, she received M. Sc. degree in Control Systems from Imperial College London, U.K. She is currently pursuing the Ph.D. degree in Control Engineering at Imperial College London, U.K. Her research interests include finitetime system identification and estimation, design of fast fault detection and isolation schemes.

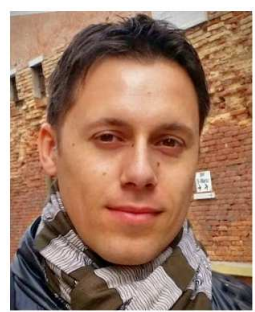

Gilberto Pin received the Laurea (M.Sc.) degree in Electrical Engineering (with honors) and the Ph.D. in Information Engineering from the University of Trieste, Italy, in 2005 and 2009, respectively. From 2009 to 2012 he was an Automation Engineer at Danieli Automation S.p.A., Italy. From 2013 to 2015 he has been a Control Systems Engineer at the R\&D Dept. of Electrolux Professional S.p.A., Italy. Since 2015, he is Energy Conversion Engineer at the Global Connectivity \& Technology Center of Electrolux Major Appliances S.p.A., Italy. He is author of several papers published in international conferences and scientific journals concerning control systems theory, signal processing methods and control applications. He is co-recipient of the IFAC Best Application Paper Prize of the Journal of Process Control, Elsevier, for the three-year period 2011-2013. His current research interests include networked control, model predictive control, advanced systems identification methods, deadbeat observers design and industrial application of advanced control techniques. Since 2012 he is Associate Editor of the Conference Editorial Board (CEB) of the IEEE Control Systems Society. He is currently serving as Associated Editor of the IEEE Trans. on Control Systems Technology.

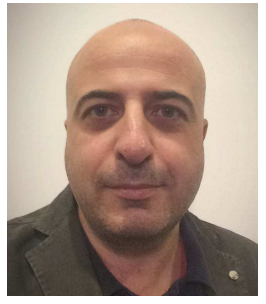

Giuseppe Fedele (M' 09) was born in Cosenza, Italy, in 1974. He received the degree (cum laude) in Computer Science engineering in 1999 and the Ph.D. degree in Computer Science and System Engineering in 2005, both from the University of Calabria, Italy. Since 2006 he has been Assistant Professor in Control Engineering at University of Calabria, Department of Informatics, Modeling, Electronics and Systems Engineering. He is a founding member of GiPStech s.r.l., a startup and Spin-off of the University of Calabria which develops novel solutions for the indoor localization and navigation problems. His current research interests include power systems, identification and filtering methods, adaptive control, adaptive algorithms for active noise and vibration control, signal processing for localization, navigation and tracking.

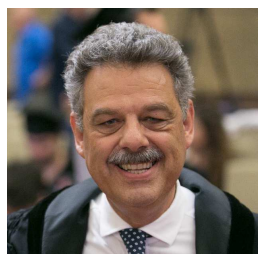

Thomas Parisini (F' 11) received the Ph.D. degree in Electronic Engineering and Computer Science in 1993 from the University of Genoa. He was with Politecnico di Milano and since 2010 he holds the Chair of Industrial Control and is Director of Research at Imperial College London. He is a Deputy Director of the KIOS Research and Innovation Centre of Excellence, University of Cyprus. Since 2001 he is also Danieli Endowed Chair of Automation Engineering with University of Trieste. In 2009-2012 he was Deputy Rector of University of Trieste. He authored or co-authored more than 280 research papers in archival journals, book chapters, and international conference proceedings. His research interests include neural-network approximations for optimal control problems, fault diagnosis for nonlinear and distributed systems, nonlinear model predictive control systems and nonlinear estimation. He is a co-recipient of the IFAC Best Application Paper Prize of the Journal of Process Control, Elsevier, for the three-year period 2011-2013 and of the 2004 Outstanding Paper Award of the IEEE Trans. on Neural Networks. He is also a recipient of the 2007 IEEE Distinguished Member Award. In 2016 he was awarded as Principal Investigator at Imperial of the H2020 European Union flagship Teaming Project KIOS Research and Innovation Centre of Excellence led by University of Cyprus. In 2012 he was awarded an ABB Research Grant dealing with energy-autonomous sensor networks for self-monitoring industrial environments. Thomas Parisini currently serves as Vice-President for Publications Activities of the IEEE Control Systems Society and during 2009-2016 he was the Editor-in-Chief of the IEEE Trans. on Control Systems Technology. Since 2017, he is Editor for Control Applications of Automatica. He is also the Chair of the IFAC Technical Committee on Fault Detection, Supervision \& Safety of Technical Processes - SAFEPROCESS. He was the Chair of the IEEE Control Systems Society Conference Editorial Board and a Distinguished Lecturer of the IEEE Control Systems Society . He was an elected member of the Board of Governors of the IEEE Control Systems Society and of the European Control Association (EUCA) and a member of the board of evaluators of the 7th Framework ICT Research Program of the European Union. Thomas Parisini is currently serving as an Associate Editor of the Int. J. of Control and served as Associate Editor of the IEEE Trans. on Automatic Control, of the IEEE Trans. on Neural Networks, of Automatica, and of the Int. J. of Robust and Nonlinear Control. Among other activities, he was the Program Chair of the 2008 IEEE Conference on Decision and Control andGeneral Co-Chair of the 2013 IEEE Conference on Decision and Control. Prof. Parisini is a Fellow of the IEEE and of the IFAC. 